\title{
Newly diagnosed cystic fibrosis in middle and later life
}

\author{
B HUNT, DM GEDDES \\ From the London Chest Hospital, London
}

ABSTRACT Four patients with cystic fibrosis diagnosed in middle and later life are presented. All had chronic bronchopulmonary infection with a high sweat sodium concentration, and chest radiographic evidence of upper zone bronchiectasis. Two patients had pancreatic dysfunction. Sputum culture grew mucoid Pseudomonas aeruginosa in three patients and Haemophilus inftuenzae in one. Ages at diagnosis were 63, 42, 40, and 35 years. These patients confirm the possibility of occasional longevity in cystic fibrosis and emphasise the need to consider the diagnosis at all ages. They also provide encouragement for younger patients.

The prognosis of cystic fibrosis has improved considerably over the post three decades. Previously death in the first year of life was common and very few patients with cystic fibrosis lived for more than 10 years. With the advent of systematic treatment with antibiotics and physiotherapy this outlook has changed and up to $75 \%$ of affected individuals are now reaching adult life. There are, however, some adult patients with cystic fibrosis with very mild, slowly progressive disease whose survival must be a reflection of the variable natural history of cystic fibrosis rather than the effects of treatment. In the past such patients are likely to have escaped diagnosis and some may be attending chest clinics unrecognised, or may still be undiagnosed in the community; the size of this population is unknown. The diagnosis of cystic fibrosis over the age of 30 years has been reported only rarely, although there are now many treated patients reaching this age. ${ }^{2}$ Only a handful of patients have been diagnosed after the age of $40^{3-7}$; the most recent report ${ }^{6}$ is of a 69 year old man with an abnormal response to the sweat test, abnormal pancreatic function, and chronic obstructive airways disease. A further four patients with cystic fibrosis diagnosed in middle and late adult life are presented here. Three of the patients were over 40 at diagnosis and one, a 63 year old, is the oldest woman with cystic fibrosis to be reported.

Address for reprint requests: Dr DM Geddes, London Chest Hospital, London E2 9JX.

Accepted 28 September 1984

\section{Method}

Each patient had a sweat sodium concentration measured by the pilocarpine iontophoresis method of Gibson and Cook. ${ }^{8}$ It was done on both arms on at least two occasions and at least $100 \mathrm{mg}$ of sweat was collected each time by a nursing sister experienced in the technique. Three patients had a suppression test using fludrocortisone ${ }^{4}$; they were given $5 \mathrm{mg}$ of $9-\alpha$-fludrocortisone on two successive nights before sweat sodium analysis.

Exocrine pancreatic function was assessed, firstly, by faecal fat collection for three to five days while a normal diet was being taken and, secondly by the $N$-benzoyl-l-tyrosyl- $p$-aminobenzoic acid test. ${ }^{10}$ Half a gram of this synthetic peptide is given with $5 \mu \mathrm{g}{ }^{14} \mathrm{C}$ labelled $p$-aminobenzoic acid (PABA) together with $25 \mathrm{~g}$ of casein in $500 \mathrm{ml}$ of water. PABA is released from the synthetic peptide in the duodenum in proportion to the amount of chymotrypsin available and then absorbed and excreted in the urine. Urine is collected for six hours and the amount of PABA excreted is expressed as a percentage of the ${ }^{14} \mathrm{C}$ PABA collected. Phenacetin, paracetamol, sulphonamides, nitrazepam, and foodstuffs such as prunes and cranberries were excluded from the diet as they invalidate the test.

\section{Results}

The case histories are described below and the principal clinical details and results of investigations are summarised in the table. No consistent abnormalities emerged from routine haematological and biochemical testing.

All patients had had recurrent chest infections 
Details of the patients

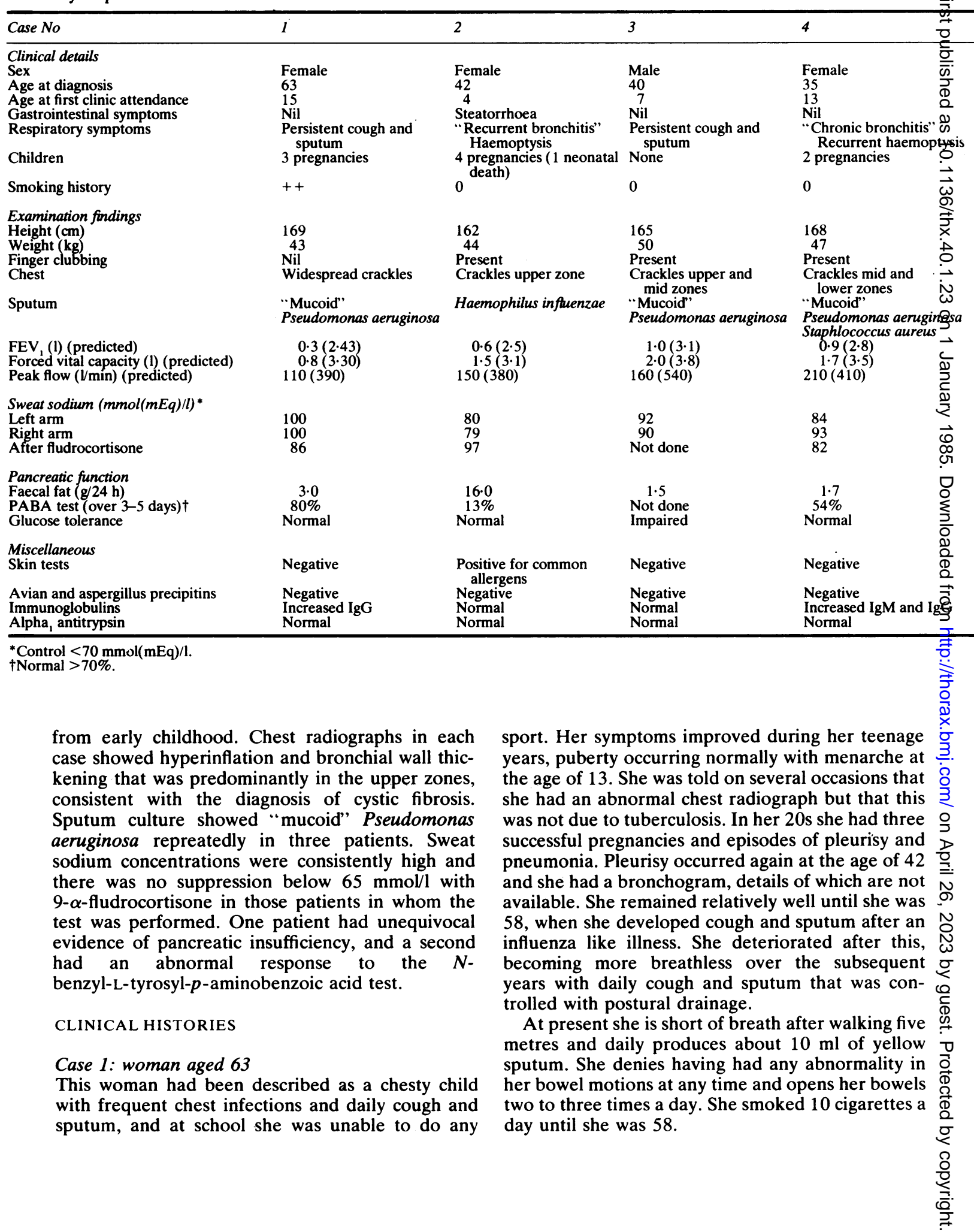


Case 2: woman aged 42

This woman had daily cough and sputum production from the age of 2 , with frequent exacerbations resulting in many hospital admissions as a child. A shadow on her chest radiograph led to treatment with isoniazid and aminosalicylic acid (PAS) for one year. From the age of 5 she had frequent bowel motions and steatorrhoea and was started on a gluten free diet, to which she adhered incompletely. By her 20s she was taking a normal diet with no worsening of her symptoms. Over the last few years she had again had frequent pale stools, which were sometimes difficult to flush away; and she had been losing weight steadily for two years.

She had four pregnancies; the second child died in the neonatal period. After the birth of the fourth child when she was 35 her sputum production increased to a cupful a day with intermittent haemoptysis. At present she can walk up one flight of stairs and can cope with the housework. There is no family history of chest disease and she is a nonsmoker.

On examination she was thin. After a normal jejunal biopsy specimen was obtained she was treated with pancreatic extract (Pancrex V). Her bowel motions became normal and she gained $4 \mathrm{~kg}$ in weight.

\section{Case 3: man aged 40}

As a child this man had had frequent chest infections and at the age of 20 he was told that he had "bronchitis." Subsequently he had recurrent chest infections and a gradual increase in sputum volume. In 1966 a bronchogram showed bilateral apical and right middle lobe bronchiectasis. He continues to have courses of antibiotics and his exertional breathlessness is increasing.

\section{Case 4: woman aged 35}

This woman had "pneumonia" at the age of 2 and had cough and sputum intermittently throughout her childhood; they became persistent after the age of 10 . During her teens and early 20 s she had persistent cough and sputum, which resulted in further investigation. She had two courses of antituberculous treatment despite negative sputum cultures and a negative response to the Mantoux test. Sputum persistently grew Pseudomonas aeruginosa and Haemophilus infuenzae had also been cultured. She continues to have daily sputum production - up to half a cupful a day-and episodes of haemoptysis, necessitating frequent treatment with antibiotics; and she is short of breath on climbing stairs. Past illnesses include a thyroidectomy at the age of 28 for tracheal compression. She has had two successful singleton pregnancies. There is no family history of chest disease and she has never smoked.

\section{Discussion}

The first reported case of cystic fibrosis to be newly diagnosed over the age of 20 was by Hellerstein in $1946^{11}$; this was of an American Negro who died at the age of 35 . Subsequent case reports of cystic fibrosis diagnosed in adulthood are rare; there are still few published reports of patients newly diagnosed over the age of 30 and only a handful of patients older than 40. The oldest reported survivors with cystic fibrosis are a 52 year old American woman and a 69 year old Norwegian man. ${ }^{4-6}$

The diagnosis of cystic fibrosis depends on several features, including chronic bronchopulmonary infection, pancreatic insufficiency, a high sweat sodium concentration, and a family history of cystic fibrosis; not all patients have all these and in particular pancreatic insufficiency is absent in up to $15 \% .^{12}$ Stern et al have recently suggested modifications dividing the diagnostic criteria into major and minor criteria, following from their work on obstructive azoospermia. ${ }^{13}$ The diagnosis is established by the presence of one major with two minor or two major findings; the two criteria for diagnosis must include different organ systems. All our patients fulfill these criteria.

A sweat sodium concentration of over $60 \mathrm{mmol}$ $(\mathrm{mEq}) / \mathrm{l}$ in a child is usually considered diagnostic of cystic fibrosis, but sweat sodium concentrations tend to increase with age and show wide variability between individuals. ${ }^{14}$ There are several series reporting sweat sodium concentrations in normal people from older age groups. ${ }^{1516}$ In McKendrick's series of 110 normal adults only five had a concentration greater than $80 \mathrm{mmol} / 1 .{ }^{14}$ Recent work by Hodson et al suggested that cystic fibrosis should be diagnosed in adults when two measurements of sweat sodium are above $70 \mathrm{mmol} / \mathrm{l}$, and diagnostic accuracy was improved in borderline cases by a suppression test using fludrocortisone." All our patients had sweat sodium concentrations greater than $80 \mathrm{mmol} / \mathrm{l}$ and no suppression below $65 \mathrm{mmol} / \mathrm{l}$ with 9$\alpha$-fludrocortisone occurred in the three patients in whom the test was done. When the baseline sweat sodium concentration was plotted against the lowest concentration all results were well above the best discriminatory line as described by Hodson et al. ${ }^{9}$

The clinical pattern of chest disease in these patients was typical of cystic fibrosis. All had a long history of respiratory infections that started in childhood, the chest radiographs showed changes of bronchiectasis predominantly affecting the upper zones, and bacteria cultured from the sputum were also typical. Exocrine pancreatic insufficiency was 
demonstrated in two of our patients; another had an abnormal response to the glucose tolerance test. In di Sant 'Agnese's series of adult patients with cystic fibrosis $^{2} 95 \%$ had pancreatic insufficiency but, in contrast to the younger patients, it was seldom symptomatic and in many cases dietary restrictions were unnecessary. Chronic pulmonary diseases has been the predominant cause of morbidity in our patients. No patient had a family history of cystic fibrosis but this is only a minor diagnostic criterion and is usually not found.

The most interesting feature of these four patients is their longevity. While this may reflect only the mild end of a continuous spectrum of clinical disease, there are other possible explanations, including a mild variant of cystic fibrosis and an improved prognosis in those without pancreatic insufficiency. There is little evidence to support genetic heterogeneity in cystic fibrosis, although it is possible that other genes modify the clinical expression of the disorder. The absence of pancreatic disease resulting in better nutrition may protect the chest from rapid deterioration seen in the most severely affected. At present the relative contributions of chest and pancreatic dysfunction to the clinical expression of cystic fibrosis is uncertain.

All our patients had been attending specialist clinics for some years before the diagnosis of cystic fibrosis was made. This suggests that there must be many others in the clinic population who are as yet undiagnosed. Wood ${ }^{17}$ looked at 24 emphysematous patients, of whom five had an abnormal sweat chloride concentration and impaired absorption of neutral fat; the mean chloride concentrations, however, were only on the borderline of abnormality. Karlish and Tarosky ${ }^{18}$ made a search for cystic fibrosis in adults attending a chronic lung disease clinic and found that 11 of 33 adults previously diagnosed as having bronchitis, emphysema, asthma, or bronchiectasis had abnormally high sweat electrolyte concentrations that were consistent with cystic fibrosis and some also had abnormal bronchograms. It is uncertain how many, if any, of the patients from those two studies had cystic fibrosis. The patients we report have excellent positive evidence of cystic fibrosis and emphasise the need to consider this diagnosis in any patient with persistent unexplained chest infections, particularly if combined with malabsorption. A sweat test should form part of the diagnostic investigation regardless of the age of the patient.

Finally, these patients are important for two other reasons. Firstly, they confirm that longevity is poss- ible with cystic fibrosis, which should provide encouragement for younger patients; and secondly, the identification of long survivors may well contribute to an understanding of the aetiology and pathophysiology of the disease.

\section{References}

1 Schwachman H, Kowalsi M, Khlaw KT. Cystic fibrosis: a new outlook. Medicine (Baltimore) 1977;56:129-46.

2 Sant Agnese PA di, Davis PB. Cystic fibrosis in Adults. Am J Med 1979;66:121-32.

3 Marks BL, Anderson CM. Fibrocystic disease of the pancreas in a man aged 46. Lancet 1960;i:365.

4 Cece JD. Pancreatic Cystic Fibrosis in an Adult. J A M A 1962;181:31.

5 Scott-Sanders J, Pryor TD, IVedel MK. Prolonged Survival in an Adult with Cystic Fibrosis. Chest 1977;2:126.

6 Evensen SA. Old man with chronic obstructive pulmonary disease, pancreatic insufficiency and elevated sweat electrolytes. Acta Med Scand 1981;209: 141-3.

7 Spencer Jones J. Adult cystic fibrosis, fatty replacement of the pancreas in woman aged $47 . \mathrm{BrJ}$ Dis Chest $1970 ; 64: 25$.

8 Gibson LE, Cooke RE. A test for concentration of electrolytes in sweat in cystic fibrosis of the pancreas utilising pilocarpine by iontophoresis. Pediatrics 1959;23:545-9.

9 Hodson ME, Beldow I, Power R, Duncan SR, Bamber $\mathrm{M}$, Batten JC. Sweat test to diagnose cystic fibrosis in adults. $\mathrm{Br}$ Med J 1983;286:1381-3.

10 Arranitakis C, Greenberger NJ. Diagnosis of pancreatic disease by a synthetic peptide-a new test of exocrine pancreatic function. Lancet 1976;i:663-6.

11 Hellerstein HK. Cystic fibrosis of the pancreas in an adult. Ohio Med J 1946;42:616.

12 Editorial: Diagnosis of cystic fibrosis. Lancet 1982;ii: 1196-7.

13 Stern RC, Boat TA, Doershuck CF. Obstructive azoospermia as a diagnostic criterion for the cystic fibrosis syndrome. Lancet 1982;i:1401-4.

14 McKendrick T. Sweat sodium levels in normal subjects in fibrocystic patients and their relatives, and in chronic bronchitis patients. Lancet 1962;i: 183-6.

15 Anderson MA, Freeman M. Sweat test results in normal persons of different ages compared with families with fibrocystic disease of the pancreas. Arch Dis Child 1960;35:581-7.

16 Lobeck CC, Huebner D. Effects of age, sex and cystic fibrosis on the sodium and potassium content of human sweat. Pediatrics 1962;30:172.

17 Wood JA, Fisherman AP, Reetntsmak, Barker AG, di Sante 'Agnese PA. A comparison of sweat chloride and intestinal fat absorption in chronic obstructive pulmonary emphysema and fibrocystic disease of the pancreas. N Engl J Med 1959;260:951-7.

18 Karlish A, Tarosky AL. Mucoviscidosis as a factor in chronic lung disease in adults. Lancet 1960;ii:514. 